\section{Fe-Based Amorphous Alloy Rods with 12-mm Diameter Developed}

Amorphous Fe-based alloys show several properties that are superior to those of their crystalline counterparts and structural steels. They have higher strength and hardness, superior corrosion resistance, and better magnetic properties, depending on the application. However, because of their limited glass-forming ability, until now it has only been possible to form rods with maximum dimensions in the range of $4 \mathrm{~mm}$. If these alloys are to be used for structural applications, it is necessary to form much larger blocks of the material. It was recently shown that the addition of yttrium improves the glass-formability of Fe-based alloys. The larger $\mathrm{Y}$ atoms induce atomic-level stress that was shown to retard crystal phase formation, with the system left in a vitrified state on cooling from the melt.

Two new studies have now used this principle to develop structural amorphous Fe alloys with maximum thickness diameters approaching $12 \mathrm{~mm}$. V. Ponnambalam, S.J. Poon, and G.J. Shiflet of the University of Virginia reported the development of Fe-based bulk metallic glasses with thicknesses of more than $1 \mathrm{~cm}$ by alloying $\mathrm{Y}$ and Er in the May 2004 issue of the Journal of Materials Research (p. 1320). Z.P. Lu, C.T. Liu, J.R. Thompson, and W.D. Porter of Oak Ridge National Laboratory reported their work on developing amorphous Fe alloy rods with large diameters in the June 18, 2004, issue of Physical Review Letters (245503). The two studies go a long way in forging a path toward the ultimate goal of using amorphous Fe-based alloys for structural applications.

Poon and colleagues used an Fe-Cr-MoC-B alloy system with $2 \%$ yttrium and erbium, a lanthanide, in the alloy (see Figure 1). The Y/Er-to-Fe atomic size ratio is about 1.4, which is close to the largest practically attainable value in Fe-based alloys. Samples were prepared by injecting the molten alloy into a copper mold under optimized casting conditions. Rods of up to $12 \mathrm{~mm}$ in diameter were found to remain amorphous, as confirmed by $\mathrm{x}$-ray diffraction (XRD). The Young's modulus of the material was $200 \mathrm{GPa}$. with the bulk modulus in the range of $180 \mathrm{GPa}$. Tensile strength, based on microhardness measurements, was $\sim 4 \mathrm{GPa}$, with hardness values of $\sim 13 \mathrm{GPa}$. The research team hypothesized that the metastable $\mathrm{Fe}_{23} \mathrm{C}_{6}$ phase that forms upon devitrification of $\mathrm{Fe}-\mathrm{Cr}-$ Mo-C-B becomes less stable when alloyed with $\mathrm{Y}$ and Er. Lanthanide elements other than Er, such as Dy, Yb, and Gd, also yielded similar amorphous Fe alloys.

For their investigations, Lu and col-

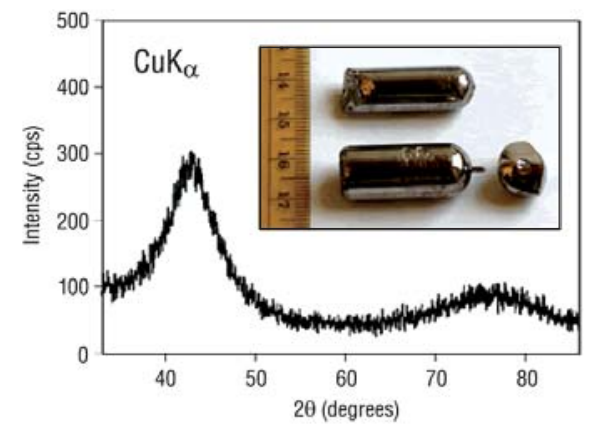

Figure 1. X-ray diffraction pattern for an $\mathrm{Fe}-\mathrm{Cr}-\mathrm{Mo}-\mathrm{Er}-\mathrm{C}-\mathrm{B}$ sample, revealing its amorphous nature. The inset shows a photo of (top) 10-mm-diameter and (bottom) 12-mm-diameter glassy rods as well as a small segment obtained from a 12-mm-diameter glassy rod. Reproduced with permission from the Journal of Materials Research.

leagues used a Fe-Cr-Co-Mo-Mn-C-B system with $1.5 \% \mathrm{Y}$ added. Alloy ingots were obtained by arc-melting a mixture of the metals and elements, which were then remelted and drop-cast into a copper mold. XRD and optical microscopy were used to confirm that the material was amorphous. It was shown that $12-\mathrm{mm}-$ diameter rods could be obtained for a certain compositional range in a pseudoternary phase diagram. When cast without $Y$, the material immediately showed crystalline characteristics. The Young's modulus of the new amorphous material was more than double that of bulk amorphous Pd-Cu-Ni-P metallic glass, and nanoindentation hardness values were more than triple. The materials were paramagnetic at room temperature and became ferromagnetic at cryogenic temperatures. The research team hypothesized that the superior glass-forming ability of the new alloys is due to lower eutectic temperatures and a shift of the composition to the eutectic, wherein glass formation is thermodynamically favored. Also, addition of $\mathrm{Y}$ retarded the nucleation and growth of Fe carbides, the primary phase, on cooling from the melt. The yttrium addition destabilized competing crystalline phases while stabilizing liquid phases.

Both studies have demonstrated that the addition of a minor alloying element is extremely effective in promoting bulk metallic glass formation in Fe alloys. These studies suggest that in the future such materials could potentially replace conventional high-strength steels in structural and other applications. In a separate study, William L. Johnson and colleagues at the California Institute of
Technology reported in the June 18 issue of Physical Review Letters (245504) that the addition of $\mathrm{Y}$ to a $\mathrm{Cu}-\mathrm{Zr}-\mathrm{Al}$ system was also shown to yield the formation of amorphous copper alloys with dimensions of up to $12 \mathrm{~mm}$.

GOPAL RAO

\section{Conductance in Single DNA Molecules Directly Measured}

The direct measurement of conductance in single DNA molecules is important for the study of charge transport, which is important in human health issues, and biosensor applications. Although DNA conductance measurements made in recent years show characteristics varying from insulator to superconductor, several requirements for reliable measurements have been made clear: that electronic coupling between DNA molecules and electrodes be reproducible; that a signature is discovered that identifies the conductance with a single DNA molecule; and that the measurement be performed in an aqueous buffer in order to preserve the native conformation of the DNA. N.J. Tao and coworkers at Arizona State University have met these requirements and have directly measured the conductance of single DNA molecules (with varying length and sequence) covalently bonded to electrodes in aqueous solution.

As reported in the June 9 issue of Nano Letters (p. 1105), the researchers measured the conductance of two series of DNA sequences: $(\mathrm{GC})_{n}$, where $n=4-7$; and CGCG(AT) ${ }_{m}$, CGCG, where $m=0,1,2$. Both series are self-complementary and spontaneously form stable B-form helices in solution, which is typical of most native DNA. Both DNA series are capped at each end with $-\mathrm{CH}_{2} \mathrm{CH}_{2} \mathrm{CH}_{2} \mathrm{SH}$ groups. The researchers formed molecular junctions between a Au electrode and a Au tip of a scanning tunneling microscope (STM) that was insulated over most of its area to eliminate unwanted current leaks. In a buffer solution containing $3 \mu \mathrm{M}$ DNA, the STM tip was repeatedly moved into and out of contact with the electrode. Once the STM tip made contact with the electrode, a piezoelectric transducer was activated and pulled the electrode out of contact with the tip. DNA molecules then bridged the gap between the STM tip and the electrode.

Molecular junction formation results in a series of steps in a plot of conductance as a function of tip-electrode separation. For $(\mathrm{GC})_{4}$, the steps are approximately multiples of a fundamental conductance, $1.3 \times$ $10^{-3} G_{0}$ (where $G_{0}=2 \mathrm{e}^{2} / \mathrm{h} \approx 77 \mu \mathrm{S}$, which is on the order of the conductance of a single gold atom connecting the two electrodes). The researchers attributed each step in the 